\title{
Disentanglement of Surface and Bulk Rashba Spin Splittings in Noncentrosymmetric BiTeI
}

\author{
Gabriel Landolt, ${ }^{1,2}$ Sergey V. Eremeev, ${ }^{3,4}$ Yury M. Koroteev, ${ }^{3,4}$ Bartosz Slomski, ${ }^{1,2}$ Stefan Muff, ${ }^{1}$ Titus Neupert, ${ }^{2}$ \\ Masaki Kobayashi, ${ }^{2}$ Vladimir N. Strocov, ${ }^{2}$ Thorsten Schmitt, ${ }^{2}$ Ziya S. Aliev, ${ }^{5}$ Mahammad B. Babanly, ${ }^{5}$ \\ Imamaddin R. Amiraslanov, ${ }^{6}$ Evgueni V. Chulkov, ${ }^{7}$ Jürg Osterwalder, ${ }^{1}$ and J. Hugo Dil ${ }^{1,2}$ \\ ${ }^{1}$ Physik-Institut, Universität Zürich, Winterthurerstrasse 190, CH-8057 Zürich, Switzerland \\ ${ }^{2}$ Swiss Light Source, Paul Scherrer Institut, CH-5232 Villigen, Switzerland \\ ${ }^{3}$ Institute of Strength Physics and Materials Science, Russian Academy of Sciences, \\ Siberian Branch, Akademicheskiy prospekt 2/4, Tomsk, 634021, Russia \\ ${ }^{4}$ Tomsk State University, Tomsk, 634050, Russia \\ ${ }^{5}$ General and Inorganic Chemistry Department, Baku State University, Baku, AZ1148, Azerbaijan \\ ${ }^{6}$ Institute of Physics, Azerbaijan National Academy of Science, Baku, AZ1143, Azerbaijan \\ ${ }^{7}$ Donostia International Physics Center (DIPS) and CFM, Centro Mixto CSIC-UPV/EHU, Departamento de Física de Materiales, \\ UPV/EHU, 20080 San Sebastián, Spain \\ (Received 10 April 2012; published 13 September 2012)
}

\begin{abstract}
BiTeI has a layered and noncentrosymmetric structure where strong spin-orbit interaction leads to a giant Rashba spin splitting in the bulk bands. We present direct measurements of the bulk band structure obtained with soft x-ray angle-resolved photoemission (ARPES), revealing the three-dimensional Fermi surface. The observed spindle torus shape bears the potential for a topological transition in the bulk by hole doping. Moreover, the bulk electronic structure is clearly disentangled from the two-dimensional surface electronic structure by means of high-resolution and spin-resolved ARPES measurements in the ultraviolet regime. All findings are supported by ab initio calculations.
\end{abstract}

DOI: 10.1103/PhysRevLett.109.116403

PACS numbers: 71.20.Nr, 71.70.Ej, 79.60.Bm

In materials without inversion symmetry, the spin degeneracy of the band structure can be lifted by a momentum-dependent spin-orbit interaction (SOI) [1,2]. This effect is crucial to several recent discoveries in condensed matter physics, such as the two-dimensional (2D) Dirac surface states of $\mathbb{Z}_{2}$ topological insulators [3] or the formation of Majorana fermion bound states at the end of one-dimensional (1D) superconducting InAs wires [4]. Comparably little attention has, however, been paid to the intrinsically three-dimensional (3D) effects of Rashba spin-orbit coupling in metals. In this Letter, we map out the 3D band structure of a system without bulk inversion symmetry and with strong SOI by the example of BiTeI. We argue that it has the potential to serve as a platform for exciting theoretical and experimental developments, due to its complex torus-shaped Fermi surface.

The existence of giant Rashba splitting in BiTeI has been recently reported, predicted by $a b$ initio calculations and observed as a largely spin-split two-dimensional state at the surface by means of angle-resolved photoemission (ARPES) and spin-resolved ARPES by Ishizaka et al. [5]. Theoretical work based on the perturbative $\mathbf{k} \cdot \mathbf{p}$ formalism linked the unusually large spin splitting in BiTeI to the negative crystal field splitting of the top valence bands [6]. Optical transition measurements [7] are in accordance with the giant bulk spin splitting of the gap defining valence and conduction bands predicted by first principle calculations [5,6]. In addition, it was shown in recent theoretical work that BiTeI can become a topological insulator under action of hydrostatic pressure [8] and, thus, is closely related to noncentrosymmetric topological superconductors.

In this Letter, direct measurements of the bulk band dispersion and the spindle torus shape of its Fermi surface are presented, using angle-resolved photoemission spectroscopy in the ultraviolet (UVARPES) and soft x-ray regime (SXARPES), as well as spin-resolved ARPES (SARPES). Moreover, we establish that the twodimensional Rashba-split state observed previously [5] is not a bulk-derived quantum well state (QWS) but rather a surface state based on (i) the first-time simultaneous observation of the $k_{z}$-dispersing bulk band measured with SXARPES, (ii) comparison with ab initio calculations, and (iii) the observed constant spin structure of the surface state upon inversion of the crystal out-of-plane axis. A clear distinction from the bulk band structure and the understanding of the origin and the properties of the surface state are crucial for future spintronic applications and tuning of the transport properties.

All measurements were performed at the Swiss Light Source of the Paul Scherrer Institut. The SARPES data were measured with the Mott polarimeters at the COPHEE end station [9] of the Surface and Interface Spectroscopy beam line at a photon energy of $24 \mathrm{eV}$. The spin-integrated data at photon energies of $20-63 \mathrm{eV}$ were taken at the highresolution ARPES end station at the same beam line. The soft $\mathrm{x}$-ray ARPES data were taken at the ADRESS beam line at photon energies of 310-850 eV. All spin-integrated 
measurements were performed at a sample temperature of $11 \mathrm{~K}$ and a base pressure lower than $10^{-10}$ mbar. The SARPES data were taken at $20 \mathrm{~K}$.

For structural optimization and electronic band calculations, we employed density functional theory (DFT) with the generalized gradient approximation of Ref. [10] for the exchange-correlation potential as implemented in VASP [11]. The interaction between the ion cores and valence electrons was described by the projector augmented-wave method [12]. The Hamiltonian contained scalar relativistic corrections, and the SOI was taken into account by the second variation method [13].

BiTeI has a hexagonal crystal structure and is built up of alternating layers of bismuth, tellurium, and iodine atoms stacked along the hexagonal axis. The continuous stacking order of the layers of the three atomic species breaks the inversion symmetry. DFT calculations show that the absence of inversion symmetry allows the strong SOI to lift the spin degeneracy of every band away from the time reversal invariant momenta $(\Gamma, A, M, L)$. In particular, the bulk conduction band minimum $(\mathrm{CBM})$ and the valence band maximum (VBM) are shifted away from the $A$ point at the Brillouin zone boundary towards the $H$ and $L$ points.

Because of the broken inversion symmetry, an untwinned BiTeI crystal has two different terminations. The weak bonding between the Te and I layers provides a natural cleaving plane; therefore, the termination layer is either purely formed of iodine or tellurium atoms, depending on the stacking order of the underlying layers. Despite the lacking inversion symmetry in the crystal structure, the bulk band structure neglecting spin is inversion symmetric due to the time reversal symmetry.

The bulk band structure of BiTeI as observed with SXARPES is presented in Figs. 1(a)-1(c). At these high photon energies, photoemission from the bulk states is dominant over surface state emission, while the latter is emphasized in the more surface sensitive UVARPES [Figs. 1(e)-1(g)]. Figure 1(a) shows a band map along $A-L$ measured at $760 \mathrm{eV}$ photon energy. In accordance with previous optical measurements, the bulk band gap is $400 \mathrm{meV}$ [7]. The 3D nature of these bands is evident from their dispersion behavior, which is shown in Fig. 1(b). Both for the valence band and the conduction band, a clear $k_{z}$ dispersion is observed. At $760 \mathrm{eV}$ photon energy, corresponding to the 16th $A$ point in the Brillouin zone, the CBM binding energy is largest and decreases away from the $A$ point and eventually disperses above the Fermi level around $800 \mathrm{eV}$ photon energy.

Figure 1(c) shows the dispersion along the $\Gamma-A$ direction, i.e., for $k_{\|}=0$, measured by sweeping photon energies from 310 to $850 \mathrm{eV}$. Because of the non-negligible photon momentum transferred to the photoelectrons at the used photon energies, the measured electron momenta have been corrected for the photon momentum projected on the particular electron momentum axis. The $k_{z}$ dispersion of the conduction band follows the periodicity of the
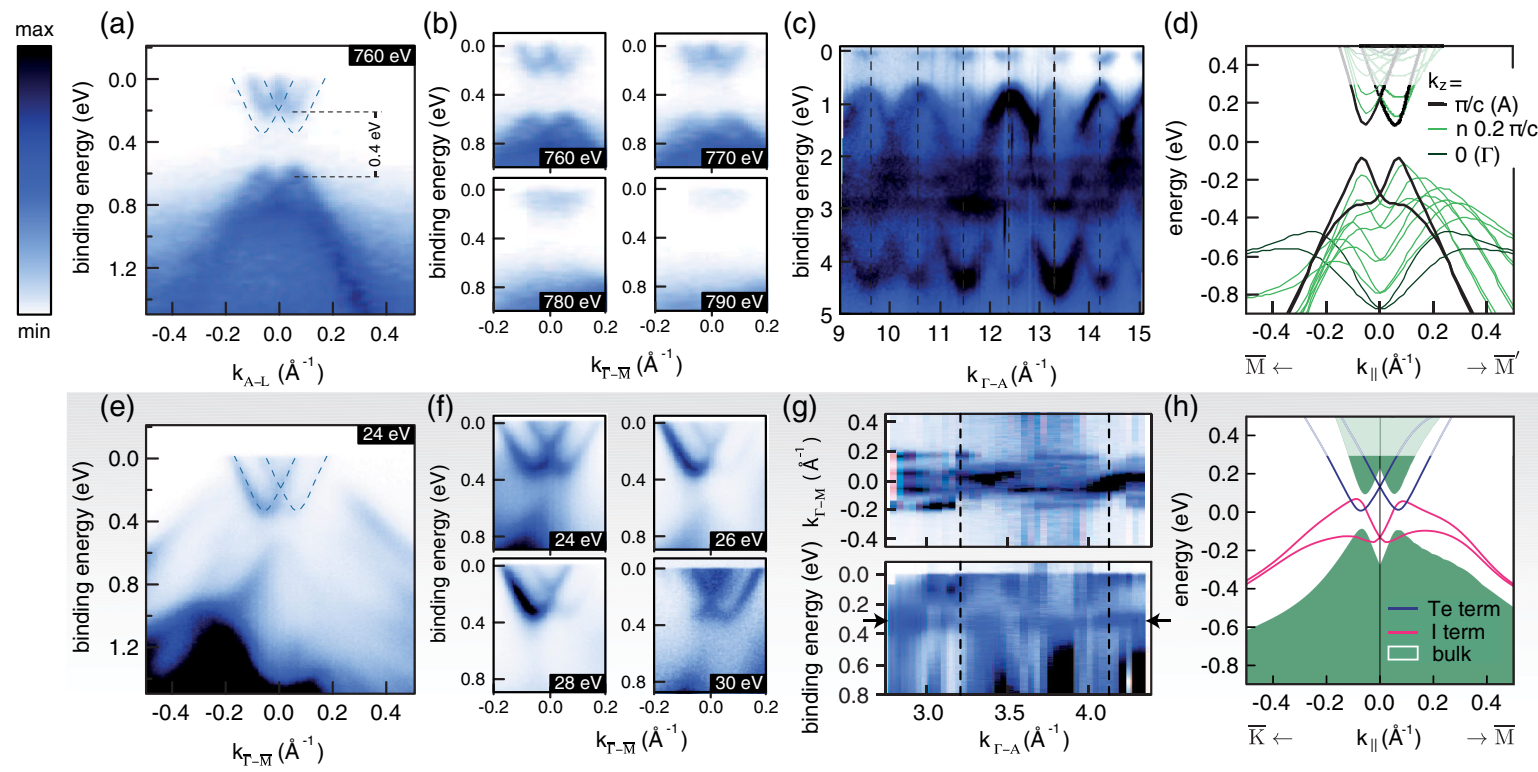

FIG. 1 (color online). (a) SXARPES band map of the bulk state at $760 \mathrm{eV}$ photon energy (16th zone boundary). (b) Band maps at 760-790 eV photon energy. (c) Band map along $\Gamma-A$ (i.e., $k_{\|}=0$ ); the dashed lines indicate the Brillouin zone boundaries. (d) DFT bulk spectra for a set of $k_{z}$ values along $\bar{M}-\bar{\Gamma}-\bar{M}^{\prime}$. The measured Fermi level is indicated by a lower intensity of the unoccupied states. (e),(f) High-resolution ARPES band map of the surface state at 24-30 eV photon energy. (g) Fermi surface map along $\bar{\Gamma}-\bar{M}$ and map of the parabola minimum at $0.06 \AA^{-1}$ as a function of photon energy (20-63 eV). Indicated are the Brillouin zone boundaries (dashed lines) and the parabola minima (arrows). (h) Slab calculations for iodine and tellurium termination. For both terminations, a surface state appears in the bulk band gap. 
valence bands, so the highest CBM binding energies occur periodically at all $A$ points $\left[k_{z}=(2 n+1) \pi / c\right.$, where $c$ is the out-of-plane lattice constant], indicated by the vertical dashed lines. The calculated $k_{z}$ dependence of the lowest conduction band and highest valence band along $\bar{M}-\bar{\Gamma}-\bar{M}^{\prime}$ reproduces these observations [Fig. 1(d)]. At the $A$ point, the calculation perfectly matches the measured spectrum at $760 \mathrm{eV}$ in Fig. 1(a), apart from a smaller calculated band gap, which is a typical problem of DFT calculations.

Our SXARPES data clearly indicate that the bulk states do disperse at the surface and are thus not quantized into quantum well states. Despite the probing depth of SXARPES being slightly larger than in the UV regime (6-8 $\AA$ at $600 \mathrm{eV}$ photon energy), it is distinctly smaller than the typical length scale for surface band bending.

Figure 2 shows the complex 3D bulk Fermi surface of BiTeI which is typical for an entire class of noncentrosymmetric materials with strong SOI and to our knowledge measured for the first time with ARPES. In the in-plane direction, the Fermi surface around the $A$ point is described by two concentric hexagonally distorted rings while it disappears around the $\Gamma$ point [14]. In the out-of-plane direction, the Fermi surface takes the form of two loops, intersecting at the $\Gamma-A$ line. The left panels of Figs. 2(a)

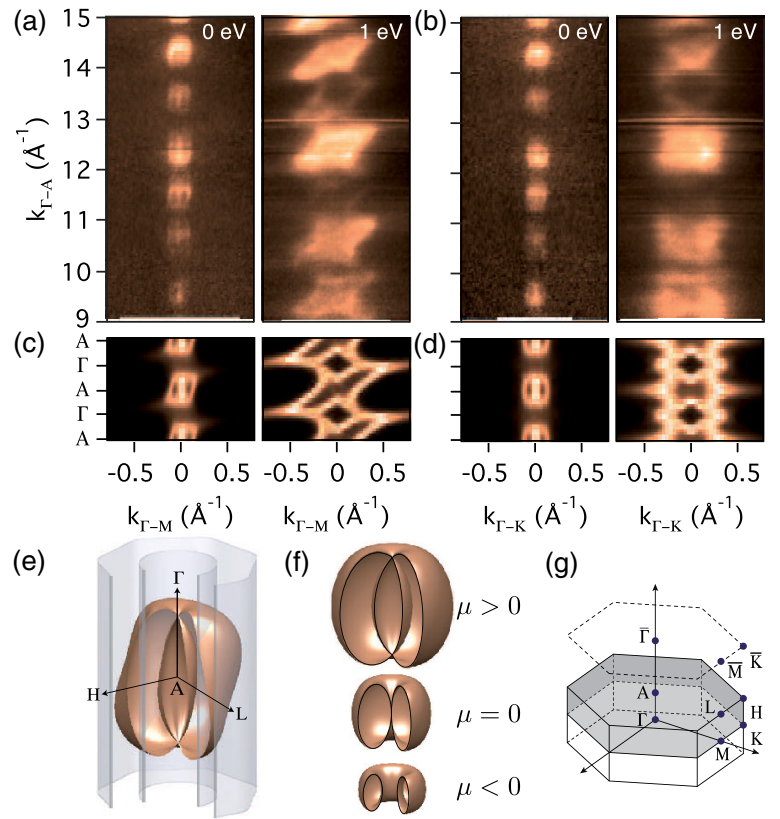

FIG. 2 (color online). (a),(b) SXARPES photon-energydependent constant binding-energy maps of the lowest bulk conduction band at the Fermi level and of the highest valence band at $1 \mathrm{eV}$ binding energy in the (a) $\Gamma-M-A-L$ and (b) $\Gamma-K-A-H$ planes. (c),(d) DFT calculations corresponding to the directions and energies in (a) and (b). (e) 3D schematic representation of the bulk Fermi surface (dark orange) and the surface state Fermi surface (light gray). (f) Topological transition of the bulk Fermi surface as a function of the chemical potential. (g) Bulk and surface Brillouin zones. and 2(b) show the measured Fermi surface in the $\Gamma-M-L-A$ and $\Gamma-K-H-A$ planes, respectively. The right panels display constant energy cuts in the same momentum planes at a binding energy of $1 \mathrm{eV}$, cutting through the valence band close to the lower gap edge.

The measured constant energy cuts are well reproduced by DFT calculations. In Figs. 2(c) and 2(d), sections through the calculated Fermi surface in the momentum planes corresponding to the measurements in Figs. 2(a) and 2(b), about $275 \mathrm{meV}$ above the CBM, are shown in the left panels, as are constant energy cuts through the valence band $485 \mathrm{meV}$ below the VBM in the right panels. The lifetime band broadening has been manually added. These data complete the picture of the 3D Rashba-type Fermi surface, taking the form of a spindle torus distorted according to the crystal symmetry and, as will be shown below, coexisting with a 2D Rashba-split surface state [Fig. 2(e)].

The UVARPES data [Figs. 1(e)-1(g)] show a state with an in-plane dispersion similar to that of the bulk conduction band state measured near the $A$ point [Fig. 1(a)] but shifted by $100 \mathrm{meV}$ to higher binding energies and exhibiting a 20\% larger momentum splitting. In contrast to Ref. [5], we interpret this state as a surface state and not a bulk-derived QWS, based on the following arguments.

First, we show the absence of dispersion of the surface state in the out-of-plane momentum $\left(k_{z}\right)$ direction through photon-energy-dependent dispersion maps, as shown in Figs. 1(e) and 1(f). Aside from strong intensity variations due to matrix element effects, the bands do not change as a function of photon energy. Taking into account that at these low photon energies half a Brillouin zone is swept by changing the photon energy by roughly $10 \mathrm{eV}$, the states can be said to not disperse in $k_{z}$ at all. There is neither a significant photon-energy dependence in the Fermi momenta nor in the parabola apex around $0.33 \mathrm{eV}$, as can be seen in the upper and lower panels in Fig. 1(g).

The origin of this state can be understood from DFT calculations for both Te- and I-terminated surfaces. To calculate the spectrum of the Te-terminated surface, we used a 24 layer slab with a free tellurium surface on one slab side and a hydrogen-passivated iodine termination on the other side. The tellurium surface hosts the Rashba-split electronlike surface state within the bulk band gap and with a dispersion that is in excellent agreement with the photoemission data. On the other hand, a holelike surface state appears at the I-terminated surface (in this case, the Teterminated side of the slab is passivated). In Fig. 1(h), we show both spin-split surface states at the Te- and Iterminated surfaces. In the UVARPES data in Fig. 1(e), a holelike state indeed crosses the gap and overlaps with the electronlike surface state in agreement with the theoretical predictions. Since on all measured samples both surface states were observed, we conclude that both surface terminations are present in domains of sizes smaller than the synchrotron light spot. This is only possible if both stacking 
orders are equally present in the sample, due to a large number of stacking faults.

We now invoke the spin structure as a further argument for the surface state interpretation. In the bulk, the stacking order breaks the inversion symmetry in the unit cell, creating the potential gradient responsible for the Rashba field. The spin structures of crystals with opposite stacking order are therefore related to each other by a reversal of the spin helicity. In a sample with both stacking orders equally present, Rashba fields of opposite directions will be balanced and no spin polarization can be measured in a spatially averaging SARPES experiment. The same would be true for a QWS formed from bulk states due to surface band bending.

Figure 3(c) gives the in-plane spin polarization measured along $\bar{\Gamma}-\bar{M}$ close to the Fermi energy [Figs. 3(a) and 3(b)] using a photon energy of $24 \mathrm{eV}$. The data reflect the structure of a large Rashba-type spin splitting with a high in-plane tangential polarization and clockwise helicity of the outer bands. The inner bands overlap strongly with the adjacent bands, resulting in a vanishing polarization amplitude near normal emission (see Refs. [14,15] for details on the data analysis). The high degree of spin polarization confirms the surface state character of the observed conduction band where the surface potential gradient produces the Rashba effect.

The ubiquitous appearance of both surface states (Te and I termination) has been taken as an indication for the presence of roughly equal amounts of both terminations on our samples [Fig. 3(d)]. In order to corroborate this fact, we have taken data from the same crystal as was used for the data in Fig. 3(c) but mounted with an inverted out-ofplane axis. Indeed, we find both surface states and the same degree of spin polarization and the same spin helicity near the Fermi energy [14].

Having disentangled the surface states from the bulk states, we now discuss the special bulk Fermi surface and its physical implications. This peculiar Fermi surface can be understood by considering free electrons with effective
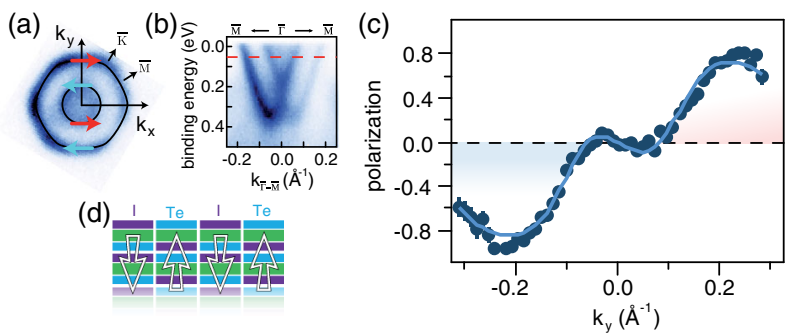

FIG. 3 (color online). (a) Surface state Fermi surface at $24 \mathrm{eV}$ photon energy. The arrows indicate the spin helicity. (b) Corresponding band map along $\bar{\Gamma}-\bar{M}$ with the dashed red line showing the binding energy of the spin-resolved measurement. (c) In-plane tangential spin polarization at the Fermi level along $\bar{\Gamma}-\bar{M}$. (d) Schematic representation of the multidomain bulk and surface. mass $m$ subject to a Rashba spin-orbit interaction of strength $\alpha$, described by the Hamiltonian

$$
H=\frac{\mathbf{k}^{2}}{2 m}+\alpha \mathbf{e}_{z} \cdot(\mathbf{k} \times \boldsymbol{\sigma})-\mu .
$$

Depending on the chemical potential $\mu$, the Fermi surface undergoes a topological transition [16]. For $-\alpha^{2} /(2 m)<$ $\mu<0$, it is a ring torus; at $\mu=0$, the hole in the torus closes; and for $\mu>0$ it becomes a spindle torus [Fig. 2(g)]. At the transition, both the number of Fermi surfaces and the genus of the remaining Fermi surface change by 1 . The toroidal $(\mu<0)$ Fermi surface encloses none of the time reversal invariant momenta, and, due to spin-momentum locking, the electronic states acquire a Berry phase of $\pi$ when transported once around the noncontractible toroidal loop that encloses the $\Gamma-A$ line.

Even though this picture does not include any lattice effects, it serves as a lowest order approximation to illustrate our results for BiTeI. Remarkably, we find that BiTeI not only shows a giant Rashba splitting in absolute terms, but also its chemical potential is found to be small compared to the spin-orbit energy scale, and thus, the measured spindle torus Fermi surface is close to the topological transition described above.

We propose that, upon hole-doping BiTeI, its Fermi surface can in fact be driven through the topological transition from the observed spindle torus to a ring torus. The resulting competition between the kinetic energy and the energy scale of the non-Abelian Rashba gauge field would render BiTeI a suitable platform for spintronics applications and an ideal playground to address questions of fundamental research. In particular, we shall illustrate the interplay of the Fermi surface topology with symmetrybreaking phenomena by the examples of superconductivity and ferromagnetism.

First, if a material with a similar bulk Fermi surface would show a superconducting instability, the topological change in the Fermi surface is predicted to drive a crossover from a Bardeen-Cooper-Schrieffer superconductor to a Bose-Einstein condensate of Cooper pairs (BCS-BEC crossover) [17]. Another interesting aspect is the expected superconducting pairing symmetry, where noncentrosymmetricity generically implies a mixture of singlet and triplet pairing: if the $p$-wave component is dominant, as is the case in $\mathrm{CePt}_{3} \mathrm{Si}$ [18], the toroidal Fermi surface has the potential to realize a fully gapped $p_{x}+i p_{y}$ superconductor in $3 \mathrm{D}$. The reason is that the ring torus has no intersection with the $\Gamma-A$ of $H-K$ lines, at which the superconducting $p_{x}+i p_{y}$ gap function vanishes.

As a second example, we consider the effect of a dopant that orders ferromagnetically. This amounts to supplementing Hamiltonian (1) by a Zeeman term $M_{z} \sigma_{z}$. In this case, if the chemical potential satisfies $\mu<M_{z}$, there arises a single 3D Fermi surface without spin degeneracy but with a nontrivial spin texture. This situation of a single nonde- 
generate Fermi surface is responsible for the potential of 1D semiconductor nanowires [4] and the 2D Dirac surface states of $\mathbb{Z}_{2}$ topological insulators [3] to host exotic physical phenomena. It would be interesting to study what effects arise from such a single nondegenerate Fermi surface in $3 \mathrm{D}$, besides its relevance for spin transport due to the reduced phase space for scattering.

To conclude, direct measurements of the bulk band dispersion of noncentrosymmetric BiTeI have been presented that confirm its giant 3D Rashba-type spin splitting, leading to the unusual spindle torus-shaped Fermi surface. We have further clarified the surface state character of the 2D Rashba-split conduction band state at the Te-terminated surface and established the relation between the 2D and 3D Fermi surfaces. The toroidal 3D Fermi surface has unique properties and may open a pathway for the realization of exotic physical systems.

We thank E. Razzoli, M. Radovic, F. Dubi, M. Kropf, and C. Hess for support during the measurements. This work was supported by the Swiss National Science Foundation.

[1] Y. A. Bychkov and E. I. Rashba, JETP Lett. 39, 78 (1984).

[2] J. H. Dil, J. Phys. Condens. Matter 21, 403001 (2009).

[3] M. Z. Hasan and C. L. Kane, Rev. Mod. Phys. 82, 3045 (2010).

[4] V. Mourik, K. Zuo, S. M. Frolov, S. R. Plissard, E. P. A. M. Bakkers, and L.P. Kouwenhoven, Science 336, 1003 (2012).

[5] K. Ishizaka et al., Nature Mater. 10, 521 (2011).
[6] M. S. Bahramy, R. Arita, and N. Nagaosa, Phys. Rev. B 84, 041202(R) (2011).

[7] J. S. Lee, G. A. H. Schober, M. S. Bahramy, H. Murakawa, Y. Onose, R. Arita, N. Nagaosa, and Y. Tokura, Phys. Rev. Lett. 107, 117401 (2011).

[8] M.S. Bahramy, R. Arita, and N. Nagaosa, Nature Commun. 3, 679 (2012).

[9] M. Hoesch, T. Greber, V. N. Petrov, M. Muntwiler, M. Hengsberger, W. Auwaerter, and J. Osterwalder, J. Electron Spectrosc. Relat. Phenom. 124, 263 (2002).

[10] J. P. Perdew, K. Burke, and M. Ernzerhof, Phys. Rev. Lett. 77, 3865 (1996).

[11] G. Kresse and J. Furthmüller, Comput. Mater. Sci. 6, 15 (1996).

[12] G. Kresse and D. Joubert, Phys. Rev. B 59, 1758 (1999).

[13] D. D. Koelling and B. N. Harmon, J. Phys. C 10, 3107 (1977).

[14] See Supplemental Material at http://link.aps.org/ supplemental/10.1103/PhysRevLett.109.116403 for further ARPES data, experimental and theoretical spin analysis, and determination of the inner potential.

[15] F. Meier, H. Dil, J. Lobo-Checa, L. Patthey, and J. Osterwalder, Phys. Rev. B 77, 165431 (2008).

[16] E. Cappelluti, C. Grimaldi, and F. Marsiglio, Phys. Rev. Lett. 98, 167002 (2007).

[17] J. P. Vyasanakere, S. Zhang, and V. B. Shenoy, Phys. Rev. B 84, 014512 (2011).

[18] E. Bauer, G. Hilscher, H. Michor, Ch. Paul, E. W. Scheidt, A. Gribanov, Yu. Seropegin, H. Noël, M. Sigrist, and P. Rogl, Phys. Rev. Lett. 92, 027003 (2004). 